\title{
Salt Intake and Cognitive Function
}

\author{
New Evidence Calls For Further Investigations
}

\author{
Giovanni Rossi • Pasquale Strazzullo
}

Published online: 26 March 2013

(C) Springer International Publishing Switzerland 2013

Reduction in dietary sodium intake is an essential component of the lifestyle modifications useful for prevention and treatment of hypertension [1]. While its efficacy has been convincingly demonstrated by randomized clinical trials, it proves difficult to implement effectively in daily clinical practice. This is partly due to an often sluggish approach by health professionals in conveying the message to their patients [2], but is also in part related to an array of psychological, social and environmental factors specific to the patient as recognised a long time ago [3].

Afsar's article in this issue of High Blood Pressure and Cardiovascular Prevention focuses on the relationship between elements of the psycho-cognitive sphere and the habitual sodium intake of hypertensive patients [4]. Among the merits of Afsar's work are the analysis of a study population of newly diagnosed, untreated hypertensive individuals and the use of recognised gold standards for the measurement of sodium intake as well as of global cognitive function, occurrence of depressive symptoms and patients' quality of sleep. Relative weaknesses are the not very large sample size, the use of a single 24-hour urine collection for the evaluation of the individual dietary salt consumption and the cross-sectional study design precluding the ascertainment of possible cause-effect associations. This notwithstanding, the main finding of the study, i.e. the inverse association of 24-hour urinary sodium excretion to cognitive function together with the lack of any relationship with depressive behaviour and to the quality of sleep, is intriguing and prompts a re-evaluation

G. Rossi · P. Strazzullo ( $\square)$

Department of Clinical Medicine and Surgery, ESH Excellence

Centre of Hypertension, "Federico II" University of Naples

Medical School, via S. Pansini, 5, 80131 Naples, Italy

e-mail: strazzul@unina.it of the results of previous studies that faced similar issues, albeit in a less comprehensive framework.

Afsar's results seem to be in keeping with a study on a geriatric population sample dating back to 1998 and showing an inverse relationship between the results of the Mini Mental State Examination and the serum sodium level, which is of course a much less reliable indicator of the habitual dietary sodium intake compared with 24-hour urinary sodium excretion [5]. They are also in agreement with the recent study by Fiocco et al., also conducted in a geriatric population, using a prospective design and showing that lower sodium consumption, estimated by a food frequency questionnaire, was associated with a lower rate of cognitive impairment over 3 years, especially in subjects with low levels of physical activity [6]. Another recent clinical trial attempted to assess whether dietary counselling aiming at increasing fruit, vegetable and fish consumption and at reducing salt intake in a sample of the geriatric population would slow down cognitive decline, but the participants unfortunately failed to achieve significant reduction in sodium chloride intake, thus preventing definitive conclusions being reached [7].

Cognitive function may be affected by a depressive condition and/or by chronic sleep disturbances [8]. It is thus noteworthy that, in the study by Afsar, no significant relationship was detected between 24-hour sodium excretion and sleep quality evaluated via the Pittsburgh Sleep Quality Index. A significant association between sleep disorders and sodium intake, estimated by food records, was recently observed in patients with heart failure in whom the frequency of apnoeas and hypopnoeas per hour of sleep was evaluated by the Apnea-Hypopnea Index score [9]. The lack of agreement between this study and the one by Afsar in hypertensive patients might be explained by the fact that the Pittsburgh Sleep Quality Index used by Afsar 
provides a global assessment of self-reported sleep quality, with only partial focus on respiratory sleep disorders, thus not allowing a quantitative assessment of sleep apnoea.

In Afsar's study, no association was apparent between levels of urinary sodium excretion and evidence of depressive symptoms. Other studies have recently examined this possible relationship with results that are again at variance with those of Afsar's report. For instance, Appelhans and co-workers reported that a better overall quality of diet and primarily reduction of salt intake were associated with lower frequency of depressive symptoms in women suffering from major depression [10]. In addition, Torres et al. showed that lower levels of urinary sodium excretion and lower urinary sodium-potassium ratio were associated with an improvement of mood in a sample of the general population [11].

Coming back to Afsar's main study finding, i.e. the association between estimated sodium intake and level of cognitive function, the possible pathophysiological mechanisms underlying this observation are presently undetermined given the largely insufficient evidence available in this respect. The author speculates that people having better cognitive function would be more aware of the risks associated with excess salt intake due to a better understanding of the educational messages released by the public health system and diffused by the media. Consequently, their attempts to reduce sodium intake would be more sustained and more often successful. According to this tentative interpretation, a worst cognitive function would be causal with respect to higher sodium consumption due to an imperfect understanding of the risks of excess salt intake. Indeed, at least two prospective studies supported possible associations between hypertension, impairment of cognitive function and development of dementia [12, 13]. This chain of events might be the consequence of vascular damage produced by high blood pressure but also by a more stressful lifestyle in turn favouring the development of hypertension. On the other hand, a stressful lifestyle has also been related to greater salt consumption per se [14] and the latter, in a meta-analysis of prospective studies, has been associated with significantly greater risk of cerebrovascular events - and then possibly of cognitive impairment secondary to vascular damage - at least in part independently of blood pressure [15].

Clearly, it would be hazardous to try to reach any conclusion given the present state of knowledge and the only general consideration that can be made, based on the few data available, is that they are compatible with a bi-directional association. High-quality evidence is definitely warranted regarding mechanistic pathways possibly linking salt intake to cognitive function and providing the 'timing' of this putative association. In particular, among the aspects deserving more attention when designing future studies, are quantification of the level of stress by the use of appropriate psychometric tests and the measurement of relevant neuroendocrine activities, standardization for the degree of literacy and the subjects' socioeconomic conditions, and proper evaluation of target organ damage.

Acknowledgements The present work was prepared in relation to the activities of the MINISAL-GIRCSI and the MENOSALEPIUSALUTE study programmes that were, respectively, part of the 2008 and 2011 CCM activities, supported by the Italian Ministry of Health. The authors have no conflicts of interest that are directly relevant to the content of this review.

\section{References}

1. Campbell N, Correa-Rotter R, Neal B, Cappuccio FP. New evidence relating to the health impact of reducing salt intake. Nutr Metab Cardiovasc Dis. 2011;2:617-9.

2. Strazzullo P, D'Elia L, Cairella G, et al. Recommending salt intake reduction to the hypertensive patient: more than just lip service. High Blood Press Cardiovasc Prev. 2012;19:59-64.

3. Luft FC, Morris CD, Weinberger MH. Compliance to a low-salt diet. Am J Clin Nutr. 1997;65(2 Suppl):698S-703S.

4. Afsar B. The relationship between cognitive function, depressive behaviour and sleep quality with 24-hour urinary sodium excretion in essential hypertensive patients. High Blood Press Cardiovasc Prev. 2013;20(1). doi:10.1007/s40292-013-0002-7.

5. Rondanelli M, Solerte SB, Ferrari E. Electrolytes and cognitive function in the elderly: relationship between serum sodium and chloride concentrations and psychometric test scores. Panminerva Med. 1998;40:191-5.

6. Fiocco AJ, Shatenstein B, Ferland G, et al. Sodium intake and physical activity impact cognitive maintenance in older adults: the NuAgeStudy. Neurobiol Aging. 2012;33:829.e21-8.

7. Kwok TC, Lam LC, Sea MM, et al. A randomized controlled trial of dietetic interventions to prevent cognitive decline in old age hostel residents. Eur J Clin Nutr. 2012; doi:10.1038/ejcn. 2012.117.

8. Sutter C, Zöllig J, Allemand M, Martin M. Sleep quality and cognitive function in healthy old age: the moderating role of subclinical depression. Neuropsychology. 2012;26:768-75.

9. Kasai T, Arcand J, Allard JP, et al. Relationship between sodium intake and sleep apnea in patients with heart failure. J Am Coll Cardiol. 2011;58:1970-4.

10. Appelhans BM, Whited MC, Schneider KL, et al. Depression severity, diet quality, and physical activity in women with obesity and depression. J Acad Nutr Diet. 2012;112:693-8.

11. Torres SJ, Nowson CA, Worsley A. Dietary electrolytes are related to mood. Br J Nutr. 2008;100:1038-45.

12. Skoog I, Lernfelt B, Landahl S, et al. 15-year longitudinal study of blood pressure and dementia. Lancet. 1996;347:1141-5.

13. Kilander L, Nyman H, Boberg M, et al. Hypertension is related to cognitive impairment: a 20-year follow-up of 999 men. Hypertension. 1998;31:780-6.

14. Henry JP. Stress, salt and hypertension. Soc Sci Med. 1988; 26(3):293-302.

15. Strazzullo P, D'Elia L, Kandala NB, Cappuccio FP. Salt intake, stroke, and cardiovascular disease: meta-analysis of prospective studies. BMJ. 2009;24(339):b4567. doi:10.1136/bmj.b4567. 